\title{
On Source Transmission over Deterministic Relay Networks
}

\author{
Soheil Mohajer *, Chao Tian ${ }^{\dagger}$, and Suhas N. Diggavi* \\ * École Polytechnique Fédéral de Lausanne, Lausanne, Switzerland. \\ Email: \{soheil.mohajer, suhas.diggavi\}@epfl.ch \\ $\dagger$ AT\&T Labs-Research, Florham Park, New Jersey, USA. \\ Email: tianeresearch.att.com
}

\begin{abstract}
Lossless transmission of a set of correlated sources over a deterministic relay network is considered for two traffic requirements. In distributed multicast, the set of sources are to be delivered to a set of destinations. The source exchange requires all the nodes with access to sources to be able to reconstruct all other sources observed at other nodes. We develop achievable regions and outer bounds for both these situations. For linear deterministic networks, these bounds coincide, yielding a characterization.
\end{abstract}

\section{INTRODUCTION}

In this paper we consider distributed transmission of correlated sources over a deterministic relay network. We examine this question in two scenarios: distributed multicast, where these correlated sources are to be delivered to several destinations; and source exchange, where a set of correlated sources are to be shared. We demonstrate achievable regions and outer bounds for these problems over networks with arbitrary topology as well as arbitrary given deterministic signal interactions. The signal interactions considered are induced by the deterministic broadcast and multiple access channels modeling wireless signal transmission.

The problem of lossless distributed transmission of correlated sources to a single receiver was introduced in the seminal work of Slepian and Wolf [9]. The direct generalization of this setup to transmission over a network represented by a specific class of directed graphs was studied in [6]. In an important paper, it was shown that source-channel separation breaks down when correlated sources are sent through a multiple access channel (MAC) [5]. This idea was explored further in [8] for the compound MAC channel. The characterization of the achievable region for deterministic broadcast-only networks (called Aref networks) was established in [10]. As far as we know, this is the first exploration of lossless distributed source transmission over general relay networks with both broadcast and multiple access signal interactions.

Clearly, given that even for simple multiple access channels, the problem remains unresolved, our achievable region does not characterize the problem for general signal interaction models. The gap between our achievability region and the outer bound is related to the joint distribution that can be

This work was supported in part by the Swiss National Science Foundation through NCCR-MICS under grant number 51NF40-111400 and by N-CRAVE under grant number FP7 ICT-2007215252. induced in the network. For the special case of the linear deterministic network, introduced in [3], we can show that indeed the achievable scheme matches the outer bound, yielding a characterization. This holds both for the distributed multicast as well as the source exchange problems.

The paper is organized as follows. We present the formal statement of the problems in Section II, and the main results in Section III. The proofs of the results are given in Sections IV and V.

\section{The Model and Problem Statement}

We consider transmission over a "wireless" network $\mathcal{G}=(\mathcal{V}, \mathcal{L})$, where $\mathcal{V}$ is the set of vertices representing the communication nodes in the relay network and $\mathcal{L}$ is the set of annotated channels between the nodes, which describe the signal interactions. Note that we term these networks "wireless" because the channels are not point-to-point links, rather, they model how the transmitted signals are superimposed and received at the receiving nodes (i.e., there is broadcast and interference). A network is called layered, if all the paths connecting nodes $u$ and $v$ have the same length, for all $u, v \in \mathcal{V}$.

The network is called deterministic if the message received at each node is a deterministic function of the messages transmitted over the edges ending at the node [3], that is,

$$
\mathbf{y}_{v}=g_{v}\left(\mathbf{x}_{u}, u \in \mathcal{I}_{v}\right),
$$

where $\mathcal{I}_{v}$ is the neighborhood of $u$, i.e, the nodes whose transmissions affect $u$. In this work, we will specialize results for linear deterministic networks, in which the functions $g_{v}$ are linear with respect to their arguments, for each node $v \in \mathcal{V}$ in the network [3]. More precisely,

$$
\mathbf{y}_{v}=\sum_{u \in \mathcal{I}_{v}} \mathbf{G}_{u, v} \mathbf{x}_{u}
$$

where $\mathbf{x} \in \mathbb{F}_{p}^{q}$, and $\mathbf{G}_{u, v} \in \mathbb{F}_{p}^{q \times q}$. Here $\mathbb{F}_{p}$ is a finite field of size $p$, and $q$ is the length of the transmission vectors.

Assume $\mathcal{S} \subseteq \mathcal{V}$ is a the set of source nodes in the network, where each of them observes the samples of its own source generated with a given rate independently over time according to some joint distribution of the sources, $\mathbb{P}\left(S_{v} ; v \in \mathcal{S}\right)$. For simplicity, we assume that the sources do not have common information in the sense of Gacs-Korner [5]. We consider the following problems. 
Distributed Multicast Problem: Consider a layered deterministic network $\mathcal{G}$, with a set of source nodes $\mathcal{S}$ and receiver nodes $\mathcal{D}$. Each receiver is interested in reconstructing the sequences observed at all the source nodes. The goal is to characterize conditions under which, the source sequences can be reliably transmitted to the receivers.

Source Exchange Problem: The source nodes wish to exchange their information, that is, each node in $\mathcal{S}$ is interested to reconstruct the source sequences generated at all the others. The nodes in the network are assumed to operate in full-duplex mode $^{1}$. The goal is to characterize all set of sources which can be reliably transmitted over the network.

\section{MAin Results}

We need the following definitions before stating the result and its proof.

Definition 1: We denote by $\Lambda(\mathcal{A} ; D)$ the set of cuts which separate a subset $\mathcal{A} \subseteq \mathcal{V}$ from a destination node $D \in \mathcal{V}$,

$$
\Lambda(\mathcal{A} ; D) \triangleq\left\{\Omega \subseteq \mathcal{V}: \mathcal{A} \subseteq \Omega, D \in \Omega^{c}\right\} .
$$

Definition 2: For a given input distribution $\left\{P\left(X_{v}\right) ; v \in\right.$ $\mathcal{V}\}$ at the nodes of the network, the min-cut between a set of source nodes, $\mathcal{S}$, and a receiver $D$ is defined as

$$
\mathcal{C}(\mathcal{S}, D)=\min _{\Omega \in \Lambda(\mathcal{S}, D)} I\left(X_{\Omega} ; Y_{\Omega^{c}} \mid X_{\Omega^{c}}\right) .
$$

Definition 3: For a given subset $\mathcal{J} \subseteq \mathcal{V}$, we denote by $\mathcal{S}_{\mathcal{J}}$ the set of all source nodes in $\mathcal{J}$, i.e., $\mathcal{S}_{\mathcal{J}}=\mathcal{S} \cap \mathcal{J}$.

The first theorem introduces an outer bound for the set of source rates can be transmitted over the network.

Theorem 1: Let correlated sources $\left(S_{1}, S_{2}, \ldots, S_{L}\right)$ are observed at nodes $\left(v_{1}, v_{2}, \ldots, v_{L}\right)$ in a layered deterministic network. Assume $b$ is the bandwidth mismatch factor of the source and the channels. Then if the set of sources can be reliably transmitted over the network, then there exist some joint input distribution $\left\{P\left(X_{v}\right) ; v \in \mathcal{V}\right\}$ for which we have

$$
H\left(\mathcal{S}_{\mathcal{J}} \mid \mathcal{S}_{\mathcal{J}^{c}}\right) \leq b \cdot \mathcal{C}(\mathcal{J}, D), \quad \forall \mathcal{J} \subseteq \mathcal{S}
$$

for each receiver node $D \in \mathcal{D}$.

The following theorem characterizes an inner bound for the the set of sources can be transmitted over a given network.

Theorem 2: Let $b$ be the common bandwidth mismatch factor of a set of correlated sources and the channel. Then the sources can be reliably transmitted to the set of receivers $\mathcal{D}$ over the network if

$$
H\left(\mathcal{S}_{\mathcal{J}} \mid \mathcal{S}_{\mathcal{J}^{c}}\right)<b \cdot \mathcal{C}(\mathcal{J}, D), \quad \forall \mathcal{J} \subseteq \mathcal{S} \quad \forall D \in \mathcal{D},
$$

for some product distribution $\prod_{v \in \mathcal{V}} P\left(X_{v}\right)$ which is independent of the sources probability distribution.

Note that all the cut-values in a linear deterministic network are simultaneously maximized by product of uniform input distributions, i.e., $\mathcal{C}(\mathcal{J}, D)=\min _{\Omega \in \Lambda(\mathcal{J}, D)} \operatorname{rank} \mathbf{G}_{\Omega, \Omega^{c}}$, where $\mathbf{G}_{\Omega, \Omega^{c}}$ denotes the transfer matrix for a particular vertex

\footnotetext{
${ }^{1}$ Note that we assume the channels from $u$ to $v$, and from $v$ to $u$ can be utilized by the network simultaneously.
}

cut $\Omega$. Therefore, we can summarize the above theorems as the following corollary.

Corollary 1: The set of sources $\mathcal{S}$ can be reliably transmitted over a linear deterministic network if and only if

$$
H\left(\mathcal{S}_{\mathcal{J}} \mid \mathcal{S}_{\mathcal{J}^{c}}\right)<b \cdot \min _{\Omega \in \Lambda(\mathcal{J}, D)} \operatorname{rank} \mathbf{G}_{\Omega, \Omega^{c}}
$$

for all source subsets $\mathcal{J} \subseteq \mathcal{S}$ and all receiver nodes $D \in \mathcal{D}$.

The following two theorems give necessary and sufficient conditions for the source exchange problem, respectively.

Theorem 3: Let correlated sources $\left(S_{1}, S_{2}, \ldots, S_{L}\right)$ are observed at nodes $\left(v_{1}, v_{2}, \ldots, v_{L}\right)$ in a deterministic network. Assume $b$ is the bandwidth mismatch factor. Then if the set of sources can be reliably exchanged between the source nodes over the network, then there exist some joint input distribution $\left\{P\left(X_{v}\right) ; v \in \mathcal{V}\right\}$ for which we have

$$
H\left(\mathcal{S}_{\mathcal{J}} \mid \mathcal{S}_{\mathcal{J}^{c}}\right) \leq b \cdot \mathcal{C}(\mathcal{J}, v), \quad \forall \mathcal{J} \subseteq \mathcal{S} .
$$

for each source node $v \in \mathcal{S}_{\mathcal{J}^{c}}$.

Theorem 4: Let $b$ be the common bandwidth mismatch of a pair of correlated sources and the channel. Then the source can be reliably exchanged over the network if

$$
H\left(\mathcal{S}_{\mathcal{J}} \mid \mathcal{S}_{\mathcal{J}^{c}}\right)<\mathcal{C}(\mathcal{J}, v), \quad \forall \mathcal{J} \subseteq \mathcal{S}, \forall v \in \mathcal{S}_{\mathcal{J}^{c}},
$$

for some product distribution $\prod_{v \in \mathcal{V}} P\left(X_{v}\right)$.

As in Corollary 1, one can show that the conditions of Theorems 3 and 4 coincide for a linear deterministic network.

Corollary 2: The set of sources $\mathcal{S}$ can be reliably exchanged among the source nodes over a linear deterministic network if and only if

$$
H\left(\mathcal{S}_{\mathcal{J}} \mid \mathcal{S}_{\mathcal{J}^{c}}\right)<b \cdot \min _{\Omega \in \Lambda(\mathcal{J}, v)} \operatorname{rank} \mathbf{G}_{\Omega, \Omega^{c}},
$$

for all $\mathcal{J} \subseteq \mathcal{S}$ and $v \in \mathcal{S}_{\mathcal{J}^{c}}$

\section{The Distributed Multicast Problem}

In this section we investigate the distributed multicast problem, and present the proofs of Theorem 1 and Theorem 2.

Proof of the Theorem 1: Let $b=n / m$ be the bandwidth mismatch and the sources can be transmitted reliably for some encoding scheme using source codewords of length $m$ and channel codewords of length $n$. For a fixed source node subset $\mathcal{J}$ and a receiver node $D \in \Omega^{c}$, consider an arbitrary cut $\Omega \in \Lambda(\mathcal{J},\{D\})$. We have

$$
\begin{aligned}
m H\left(S_{\mathcal{J}} \mid S_{\mathcal{J}^{c}}\right) & \leq m H\left(S_{\Omega} \mid S_{\Omega^{c}}\right) \stackrel{(a)}{=} H\left(S_{\Omega}^{m} \mid S_{\Omega^{c}}^{m}\right) \\
& \stackrel{(b)}{\leq} I\left(S_{\Omega}^{m} ; Y_{\Omega^{c}}^{n} \mid S_{\Omega^{c}}^{m}\right)+m \epsilon
\end{aligned}
$$

where $(a)$ holds since the source symbols at different time instances are independent and identically distributed, $(b)$ is implied by Fano's inequality and the fact that $D \in \Omega^{c}$ should be able to decode the source sequences $S_{\Omega}^{m}$. Continuing from (11), we can write 


$$
\begin{aligned}
& I\left(S_{\Omega}^{m} ; Y_{\Omega^{c}}^{n} \mid S_{\Omega^{c}}^{m}\right)=\sum_{k=1}^{n} I\left(S_{\Omega}^{m} ; Y_{\Omega^{c}}[k] \mid S_{\Omega^{c}}^{m}, Y_{\Omega^{c}}[1: k-1]\right) \\
& =\sum_{k=1}^{n}\left[H\left(Y_{\Omega^{c}}[k] \mid S_{\Omega^{c}}^{m}, Y_{\Omega^{c}}[1: k-1]\right)\right. \\
& \left.\quad-H\left(Y_{\Omega^{c}}[k] \mid S_{\Omega^{m}}^{m}, S_{\Omega^{c}}^{m}, Y_{\Omega^{c}}[1: k-1]\right)\right] \\
& \quad \stackrel{(c)}{\leq} \sum_{k=1}^{n}\left[H\left(Y_{\Omega^{c}}[k] \mid S_{\Omega^{c}}^{m}, Y_{\Omega^{c}}[1: k-1], X_{\Omega^{c}}[k]\right)\right. \\
& \left.\quad-H\left(Y_{\Omega^{c}}[k] \mid S_{\Omega}^{m}, S_{\Omega^{c}}^{m}, Y_{\Omega^{c}}[1: k-1], X_{\Omega}[k], X_{\Omega^{c}}[k]\right)\right] \\
& \quad \stackrel{(d)}{\leq} \sum_{k=1}^{n}\left[H\left(Y_{\Omega^{c}}[k] \mid X_{\Omega^{c}}[k]\right)-H\left(Y_{\Omega^{c}}[k] \mid X_{\Omega}[k], X_{\Omega^{c}}[k]\right)\right] \\
& =\sum_{k=1}^{n} I\left(X_{\Omega}[k] ; Y_{\Omega^{c}}[k] \mid X_{\Omega^{c}}[k]\right),
\end{aligned}
$$

where $Y[i: j]=(Y(i), Y(i+1), \ldots, Y(j)),(c)$ follows from the facts that $X_{\Omega^{c}}$ only depends of $\mathcal{S}_{\Omega^{c}}$ and $Y_{\Omega^{c}}[1: k-1]$, and conditioning reduces entropy and $(d)$ is implied by the fact that the outputs of the network at time $k$, only depends on its inputs $X_{\Omega}[k]$ and $X_{\Omega^{c}}[k]$. From (12) and using a standard time-sharing argument [2], one can show that

$$
I\left(S_{\Omega}^{m} ; Y_{\Omega^{c}}^{n} \mid S_{\Omega^{c}}^{m}\right) \leq n I\left(X_{\Omega} ; Y_{\Omega^{c}} \mid X_{\Omega^{c}}\right)
$$

Summarizing (11)-(13), we get the claimed inequality for any $\Omega \in \Lambda(\mathcal{J}, D)$.

Proof of the Theorem 2: For the sake of simplicity, we restrict our analysis to a network with two source nodes $\mathcal{S}=\left\{v_{1}, v_{2}\right\}$, and and two destination nodes $\mathcal{D}=\left\{D_{1}, D_{2}\right\}$. However, the same scheme can be easily extended to general case.

In this scheme, all the nodes in the network operate on blocks of length $T$ of the received signals. Let $b=n / m$, where $m$ and $n$ are integrals. Define $M=m T$ and $N=n T$. At each source node $v_{k} \in \mathcal{S}$, take the set of all typical source sequences of length $M$ as source codebook, and the index them as $\left\{s^{M}(i) ; i=1, \ldots,\left|\mathcal{I}_{\delta}\left(S_{k}\right)\right|\right\}$.

In order to utilize the source correlation for decoding, the transmission at the source nodes needs to be synchronized. This is done by buffering and delaying the transmission to transmit at the appropriate block corresponding to the layer on which the nodes are placed in the layered deterministic network. At each node $v \in \mathcal{V}$, generate a codebook of size $2^{N\left[H\left(X_{v}\right)+\epsilon\right]}$ of length $N$, each randomly picked according to $P\left(X_{v}\right)$. Also, for nodes without access to a source, fix random functions $f_{v}: \mathcal{T}_{\delta}\left(\mathcal{Y}_{v}\right) \longrightarrow \mathcal{T}_{\delta}\left(\mathcal{X}_{v}\right)$ for each node $v$, which randomly maps the received sequence (and the source codeword for the source nodes) to the codewords of the node $v, \mathbf{x}_{v}=f_{v}\left(\mathbf{y}_{v}\right)$. Here $\mathcal{T}_{\delta}(\cdot)$ denotes the typical sets. For nodes with access to a source, the random map takes into account the source observation, i.e., $f_{v}: \mathcal{T}_{\delta}\left(S_{v}\right) \times \mathcal{T}_{\delta}\left(\mathcal{Y}_{v}\right) \longrightarrow \mathcal{T}_{\delta}\left(\mathcal{X}_{v}\right)$.

Once a pair of source sequences $\left(S_{1}^{M}, S_{2}^{M}\right)$ is generated, each transmitter finds the first index $i$ (and $j$ respectively) with $s_{1}^{M}(i)=S_{1}^{M}$ (similarly for $S_{2}$ ), if it exists, or an error is declared. In the following, with slight abuse of notation, we denote the dependency of the signals received and sent by node $v$ when source sequences $S_{1}^{M}(i)$ and $S_{2}^{M}(j)$ are observed at the source nodes, by $\mathbf{y}_{v}(i, j)$ and $\mathbf{x}_{v}(i, j)$, respectively.

A destination node $D \in \mathcal{D}$ receives a sequence $\mathbf{y}_{D}$ and attempts to decode the source sequence pair sent by $v_{1}$ and $v_{2}$. An error occurs at the receiver if and only if there exist a pair $\left(i^{\prime}, j^{\prime}\right) \neq(i, j)$ such that $\left(s_{1}^{M}\left(i^{\prime}\right), s_{2}^{M}\left(j^{\prime}\right)\right) \in \mathcal{T}_{\delta}\left(S_{1}, S_{2}\right)$, and moreover, $\mathbf{y}_{D}\left(i^{\prime} \cdot j^{\prime}\right)=\mathbf{y}_{D}(i, j)$. We can define the following error events as

$$
\begin{aligned}
& \mathcal{E}_{\text {enc }}^{0}=\left\{\left(S_{1}^{M}, S_{2}^{M}\right) \notin \mathcal{T}_{\delta}\left(S_{1}, S_{2}\right)\right\} \\
& \mathcal{E}_{\text {enc }}^{k}=\left\{S_{k}^{M} \neq s_{k}^{M}(i), \forall i\right\}, \quad k=1,2 \\
& \mathcal{E}_{\text {dec }}=\bigcup_{D \in \mathcal{D}} \mathcal{E}_{\text {dec }}^{D}=\bigcup_{D \in \mathcal{D}} \bigcup_{\left(i^{\prime}, j^{\prime}\right) \neq(i, j)} \mathcal{E}_{D}\left(i^{\prime}, j^{\prime}\right)
\end{aligned}
$$

where $\mathcal{E}_{D}\left(i^{\prime}, j^{\prime}\right)$ is defined as

$$
\left\{\mathbf{y}_{D}\left(i^{\prime}, j^{\prime}\right)=\mathbf{y}_{D}(i, j),\left(s_{1}^{M}\left(i^{\prime}\right), s_{2}^{M}\left(j^{\prime}\right)\right) \in \mathcal{T}_{\delta}\left(S_{1}, S_{2}\right)\right\} .
$$

Therefore, the total error can be upper-bounded as

$$
\begin{aligned}
& \operatorname{Pr}(\text { err })=\mathbb{P}\left(\mathcal{E}_{\text {enc }}^{0} \cup \mathcal{E}_{\text {enc }}^{1} \cup \mathcal{E}_{\text {enc }}^{2} \cup \mathcal{E}_{\text {ecc }}\right) \\
& \quad \leq \mathbb{P}\left(\mathcal{E}_{\text {enc }}^{0}\right)+\mathbb{P}\left(\mathcal{E}_{\text {enc }}^{1} \mid \mathcal{E}_{\text {enc }}^{0 c}\right)+\mathbb{P}\left(\mathcal{E}_{\text {enc }}^{2} \mid \mathcal{E}_{\text {enc }}^{0 c}\right)+\mathbb{P}\left(\mathcal{E}_{\text {dec }} \mid \mathcal{E}_{\text {enc }}^{0 c}\right)
\end{aligned}
$$

It is clear by AEP that the first three terms vanish as $M$ grows. It remains to upper bound the last error probability which captures the decoding error probability. Note that

$$
\begin{aligned}
\mathbb{P}\left(\mathcal{E}_{\mathrm{dec}} \mid \mathcal{E}_{\mathrm{enc}}^{0 c}\right) \leq \sum_{D \in \mathcal{D}} \sum_{\left(i^{\prime}, j^{\prime}\right) \neq(i, j)} \mathbb{P}\left(\mathcal{E}_{D}\left(i^{\prime}, j^{\prime}\right) \mid \mathcal{E}_{\mathrm{enc}}^{0 c}\right) \\
=\sum_{D \in \mathcal{D}}\left[\sum_{\substack{i^{\prime} \neq i\\
}} \mathbb{P}\left(\mathcal{E}_{D}\left(i^{\prime}, j\right) \mid \mathcal{E}_{\text {enc }}^{0 c}\right)+\sum_{j^{\prime} \neq j} \mathbb{P}\left(\mathcal{E}_{D}\left(i, j^{\prime}\right) \mid \mathcal{E}_{\text {enc }}^{0 c}\right)\right. \\
\left.+\sum_{\substack{i^{\prime} \neq i \\
j^{\prime} \neq j}} \mathbb{P}\left(\mathcal{E}_{D}\left(i^{\prime}, j^{\prime}\right) \mid \mathcal{E}_{\text {enc }}^{0 c}\right)\right]
\end{aligned}
$$

In the following we will bound each term in the summation above. We start with $\mathcal{E}_{D}\left(i^{\prime}, j\right)$ for a fixed destination node $D$ and $i^{\prime} \neq i$. Note that we can restrict our attention to those $i^{\prime} \neq i$ for which, $s_{1}^{M}(i) \neq s_{1}^{M}\left(i^{\prime}\right)$, because otherwise decoding $\left(i^{\prime}, j\right)$ does not cause an error. This probability of error can be written as ${ }^{2}$

$$
\begin{aligned}
\operatorname{Pr}[ & \left.\mathbf{y}_{D}\left(i^{\prime}, j\right)=\mathbf{y}_{D}(i, j),\left(s_{1}^{M}\left(i^{\prime}\right), s_{2}^{M}(j)\right) \in \mathcal{T}_{\delta}\left(S_{1}, S_{2}\right) \mid \mathcal{E}_{\mathrm{enc}}^{0 c}\right] \\
= & \operatorname{Pr}\left(\left(s_{1}^{M}\left(i^{\prime}\right), s_{2}^{M}(j)\right) \in \mathcal{T}_{\delta} \mid\left(s_{1}^{M}(i), s_{2}^{M}(j)\right) \in \mathcal{T}_{\delta}\right) \\
& \cdot \operatorname{Pr}\left(\mathbf{y}_{D}\left(i^{\prime}, j\right)=\mathbf{y}_{D}(i, j) \mid \begin{array}{l}
\left.s_{1}^{M}\left(i^{\prime}\right), s_{2}^{M}(j)\right) \in \mathcal{T}_{\delta}\left(S_{1}, S_{2}\right) \\
\left.s_{1}^{M}(i), s_{2}^{M}(j)\right) \in \mathcal{T}_{\delta}\left(S_{1}, S_{2}\right)
\end{array}\right) \\
& \stackrel{(a)}{\leq} 2^{-M\left[I\left(S_{1} ; S_{2}\right)-\delta\right]} \\
& \cdot \operatorname{Pr}\left(\mathbf{y}_{D}\left(i^{\prime}, j\right)=\left.\mathbf{y}_{D}(i, j)\right|^{\left(\begin{array}{l}
\left.s_{1}^{M}\left(i^{\prime}\right), s_{2}^{M}(j)\right) \in \mathcal{J}_{\delta}\left(S_{1}, S_{2}\right) \\
\left.s_{1}^{M}(i), s_{2}^{M}(j)\right) \in \mathcal{T}_{\delta}\left(S_{1}, S_{2}\right)
\end{array}\right)}\right. \\
= & 2^{-M\left[I\left(S_{1} ; S_{2}\right)-\delta\right]} \\
& \left.\cdot \sum_{\Omega \in \Lambda\left(v_{1} ; D\right)} \operatorname{Pr}\left(\begin{array}{c}
\mathbf{y}_{\Omega^{c}}\left(i^{\prime}, j\right)=\mathbf{y}_{\Omega^{c}}(i, j) \\
\mathbf{y}_{\Omega}\left(i^{\prime}, j\right) \neq \mathbf{y}_{\Omega}(i, j)
\end{array}\right) \begin{array}{l}
\left(s_{1}^{M}\left(i^{\prime}\right), s_{2}^{M}(j)\right) \in \mathcal{T}_{\delta}\left(S_{1}, S_{2}\right) \\
\left(s_{1}^{M}(i), s_{2}^{M}(j)\right) \in \mathcal{T}_{\delta}\left(S_{1}, S_{2}\right)
\end{array}\right)
\end{aligned}
$$

\footnotetext{
${ }^{2}$ There is a minor technical issue in dealing with cuts for which there exist some nodes in $\Omega$ who are not in the flow of the sources nodes. We refer the readers to [11] for details.
} 
where $(a)$ holds because for a fixed source codebook for $S_{2}$, the number of codewords $s_{1}^{M}\left(i^{\prime}\right)$ in a random codebook for $v_{1}$ who are jointly typical with $s_{2}^{M}(j)$ is $2^{M\left[H\left(S_{1} \mid S_{2}\right)-\delta\right]}$. Dividing by the codebook size of $S_{1}$ and taking average over the randomness of the $v_{2}$ codebook, we get the right probability.

For a fixed cut $\Omega$, let $L_{\ell}(\Omega)$ and $R_{\ell}(\Omega)$ be set of nodes at layer $\ell$ in $\Omega$ and $\Omega^{c}$, respectively. We define the following events similar to [4].

- $\mathcal{L}_{\ell}$ : Event that the nodes in $L_{\ell}(\Omega)$ can distinguish between the pairs $\left(i^{\prime}, j\right)$ and $(i, j)$, i.e., $\mathbf{y}_{L_{\ell}(\Omega)}\left(i^{\prime}, j\right) \neq$ $\mathbf{y}_{L_{\ell}(\Omega)}(i, j)$.

- $\mathcal{R}_{\ell}$ : Event that the nodes in $R_{\ell}(\Omega)$ can not distinguish between the pairs $\left(i^{\prime}, j\right)$ and $(i, j)$, i.e., $\mathbf{y}_{R_{\ell}(\Omega)}\left(i^{\prime}, j\right)=$ $\mathbf{y}_{R_{\ell}(\Omega)}(i, j)$.

Similar to [4], the probability in (19) can be further upper bounded by

$$
\begin{aligned}
\operatorname{Pr}\left(\begin{array}{c}
\mathbf{y}_{\Omega^{c}}\left(i^{\prime}, j\right)=\mathbf{y}_{\Omega^{c}}(i, j) \\
\mathbf{y}_{\Omega}\left(i^{\prime}, j\right) \neq \mathbf{y}_{\Omega}(i, j)
\end{array}\right. & \left(\begin{array}{c}
\left(s_{1}^{M}\left(i^{\prime}\right), s_{2}^{M}(j)\right) \in \mathcal{T}_{\delta}\left(S_{1}, S_{2}\right) \\
\left(s_{1}^{M}(i), s_{2}^{M}(j)\right) \in \mathcal{I}_{\delta}\left(S_{1}, S_{2}\right)
\end{array}\right) \\
& =\mathbb{P}\left(\mathcal{R}_{\ell}, \mathcal{L}_{\ell-1} ; \ell=2, \ldots, \ell_{D}\right) \\
& \leq \prod_{\ell=2}^{\ell_{D}} \mathbb{P}\left(\mathcal{R}_{\ell} \mid \mathcal{R}_{\ell-1}, \mathcal{L}_{\ell-1}\right)
\end{aligned}
$$

Consider the probability expression in the above product for a fixed layer $\ell$. Note that it is conditioned on $\mathcal{R}_{\ell-1}$, i.e., the nodes in $\Omega$ in layer $\ell-1$ cannot distinguish between $\mathbf{y}_{R_{\ell-1}(\Omega)}(i, j)$ and $\mathbf{y}_{R_{\ell-1}(\Omega)}\left(i^{\prime}, j\right)$, Therefore, the nodes in $R_{\ell-1}(\Omega)$ send the same signals to the nodes in the next layer in both cases, which are jointly typical with the signal received at nodes in $R_{\ell}(\Omega)$,

$$
\left(\mathbf{x}_{R_{\ell-1}(\Omega)}\left(i^{\prime}, j\right), \mathbf{y}_{R_{\ell}(\Omega)}(i, j)\right) \in \mathcal{T}_{\delta}\left(X_{R_{\ell-1}(\Omega)}, Y_{R_{\ell}(\Omega)}\right) .
$$

However, the nodes in $L_{\ell-1}(\Omega)$ have been able to distinguish between two source pairs, and therefore mapped them into output sequences $\left(\mathbf{x}_{v} ; v \in L_{\ell-1}(\Omega)\right)$ independently. The nodes in $R_{\ell}(\Omega)$ get confused only if the received signals are also jointly typical with such different transmitted signals, i.e.,

$$
\begin{aligned}
& \mathbb{P}\left(\mathcal{R}_{\ell} \mid \mathcal{R}_{\ell-1}, \mathcal{L}_{\ell-1}\right) \\
& \quad=\mathbb{P}\left(\left(\mathbf{x}_{L_{\ell-1}(\Omega)}\left(i^{\prime}, j\right), \mathbf{x}_{R_{\ell-1}(\Omega)}\left(i^{\prime}, j\right), \mathbf{y}_{R_{\ell}(\Omega)}(i . j)\right) \in \mathcal{T}_{\delta}\right) \\
& \quad \doteq 2^{-N I\left(X_{L_{\ell-1}(\Omega)} ; X_{R_{\ell-1}(\Omega)}, Y_{R_{\ell}(\Omega)}\right)} \\
& \quad \stackrel{(d)}{=} 2^{-N I\left(X_{L_{\ell-1}(\Omega)} ; Y_{R_{\ell}(\Omega)} \mid X_{R_{\ell-1}(\Omega)}\right)}
\end{aligned}
$$

where $(d)$ holds since $X_{L_{\ell-1}(\Omega)}$, and $X_{R_{\ell-1}(\Omega)}$ are independent. Replacing (22) in (20), we get

$$
\begin{aligned}
& \operatorname{Pr}\left(\begin{array}{c}
\mathbf{y}_{\Omega^{c}}\left(i^{\prime}, j\right)=\mathbf{y}_{\Omega^{c}}(i, j) \\
\mathbf{y}_{\Omega}\left(i^{\prime}, j\right) \neq \mathbf{y}_{\Omega}(i, j)
\end{array} \mid \begin{array}{c}
\left(s_{1}^{M}\left(i^{\prime}\right), s_{2}^{M}(j)\right) \in \mathcal{T}_{\delta}\left(S_{1}, S_{2}\right) \\
\left(s_{1}^{M}(i), s_{2}^{M}(j)\right) \in \mathcal{T}_{\delta}\left(S_{1}, S_{2}\right)
\end{array}\right) \\
& \leq \prod_{\ell=2}^{\ell_{D}} 2^{-N I\left(X_{L_{\ell-1}(\Omega)} ; Y_{R_{\ell}(\Omega)} \mid X_{R_{\ell-1}(\Omega)}\right)} \\
& =2^{-N \sum_{\ell=2}^{\ell D} I\left(X_{L_{\ell-1}(\Omega)} ; Y_{R_{\ell}(\Omega)} \mid X_{R_{\ell-1}(\Omega)}\right)} \\
& \stackrel{(e)}{=} 2^{-N I\left(X_{\Omega} ; Y_{\Omega^{c}} \mid X_{\Omega^{c}}\right)} \text {, }
\end{aligned}
$$

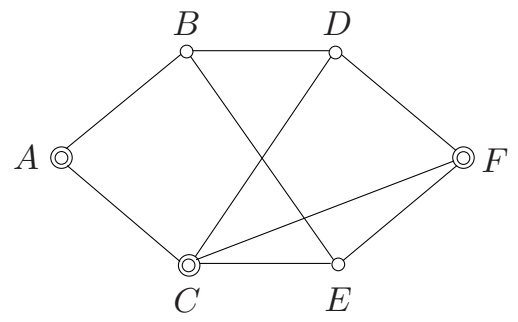

Fig. 1: The wireless network with three source nodes.

where in $(e)$ we again used the Markovian nature of the layered network. Therefore, (19) can be upper-bounded as

$$
\begin{aligned}
& \mathbb{P}\left(\mathcal{E}_{D}\left(i^{\prime}, j\right) \mid \mathcal{E}_{\text {enc }}^{0 c}\right) \leq 2^{-M\left[I\left(S_{1} ; S_{2}\right)-\delta\right]} \sum_{\Omega \in \tilde{\Lambda}\left(V_{1} ; D\right)} 2^{-N I\left(X_{\Omega} ; Y_{\Omega^{c}} \mid X_{\Omega^{c}}\right)} \\
& \quad=2^{-M\left[I\left(S_{1} ; S_{2}\right)-\delta\right]} 2^{-N \min _{\Omega \in \Lambda\left(v_{1} ; D\right)} I\left(X_{\Omega} ; Y_{\Omega^{c}} \mid X_{\Omega^{c}}\right)} .
\end{aligned}
$$

We can upper-bound the other terms in (18) in a similar way. It only remains to count the number of times each term is added in the summation, which gives us the bound in (25). It is clear that this probability goes to zero as $T \rightarrow \infty$ provided that the conditions in Theorem are satisfied.

\section{The Source Exchange Problem}

The proof of Theorem 3 is exactly the same as that of Theorem 1 in Section IV, and so we skip it here. In the following, we prove Theorem 4 by presenting and analyzing an achievable scheme.

Proof of Theorem 4: First, note that the network is not layered any more, and naturally has loops. We can use time expansion to convert an arbitrary network to a layered one for error analysis. This captures using a coding schemes involving many blocks as in [3]. Figure 2 shows the time-expanded version of the network in Figure 1.

Let $T$ be the operating time of the relay nodes, and $b=$ $n / m$, where $m$ and $n$ are integrals. Define $M=m T K$ and $N=n T K$, where $K$ is an integer denoting the number of transmission blocks. At each source node $v \in \mathcal{S}$ generate a source codebook of size $M_{v}$ with codeword length $M$, where $M_{v}=\left\lfloor 2^{M\left[H\left(S_{v}\right)+\epsilon\right]}\right\rfloor$.

Choose some product distribution for the nodes $\prod_{v \in \mathcal{V}} P\left(X_{v}\right)$, and generate random codebooks of blocklength $N$ at each node according to its distribution. In a block $k$, each node applies its random function to map whatever it has received over the last blocks, $1 \leq t \leq k-1$, to its transmit sequence in the next block.

$$
\mathbf{x}_{v}^{(k)}=f_{v}^{(k)}\left(\mathbf{y}_{v}^{(t)} ; 1 \leq t \leq k-1\right) .
$$

Similarly, such function operates on the received sequences as well as the source codeword at the source nodes. We claim that after $K$ blocks each source node $v$ is able to distinguish between different source sequences sent by the other nodes.

We restrict our analysis to the network shown in Figure 1, wherein the nodes in $\mathcal{S}=\{A, C, F\}$ are source nodes. 


$$
\begin{aligned}
& \mathbb{P}\left(\mathcal{E}_{\mathrm{dec}} \mid \mathcal{E}_{\mathrm{enc}}^{0 c}\right) \leq \sum_{D \in \mathcal{D}}[\left(2^{M\left[H\left(S_{1}\right)+\delta\right]}-1\right) 2^{-M\left[I\left(S_{1} ; S_{2}\right)-\delta\right]} 2^{-N \min _{\Omega \in \Lambda\left(v_{1} ; D\right)} I\left(X_{\Omega} ; Y_{\Omega^{c}} \mid X_{\Omega^{c}}\right)} \\
&+\left(2^{M\left[H\left(S_{2}\right)+\delta\right]}-1\right) 2^{-M\left[I\left(S_{1} ; S_{2}\right)-\delta\right]} 2^{-N \min _{\Omega \in \Lambda\left(v_{2} ; D\right)} I\left(X_{\Omega} ; Y_{\Omega^{c}} \mid X_{\Omega^{c}}\right)} \\
&\left.+\left(2^{M\left[H\left(S_{1}\right)+\delta\right]}-1\right)\left(2^{M\left[H\left(S_{2}\right)+\epsilon\right]}-1\right) 2^{-M\left[I\left(S_{1} ; S_{2}\right)-\delta\right]} 2^{-N \min _{\Omega \in \Lambda\left(\left\{v_{1}, v_{2}\right\} ; D\right)} I\left(X_{\Omega} ; Y_{\Omega^{c}} \mid X_{\Omega^{c}}\right)}\right] \\
& \leq \sum_{D \in \mathcal{D}}\left[2^{-T m\left[b \mathcal{C}\left(\left\{v_{1}\right\} ; D\right)-H\left(S_{1} \mid S_{2}\right)-\epsilon\right]}+2^{-T m\left[b \mathcal{C}\left(\left\{v_{2}\right\} ; D\right)-H\left(S_{1} \mid S_{1}\right)\right]-\epsilon}+2^{-T m\left[b \mathcal{C}\left(\left\{v_{1}, v_{2}\right\} ; D\right)-H\left(S_{1}, S_{2}\right)-\epsilon\right]}\right]
\end{aligned}
$$

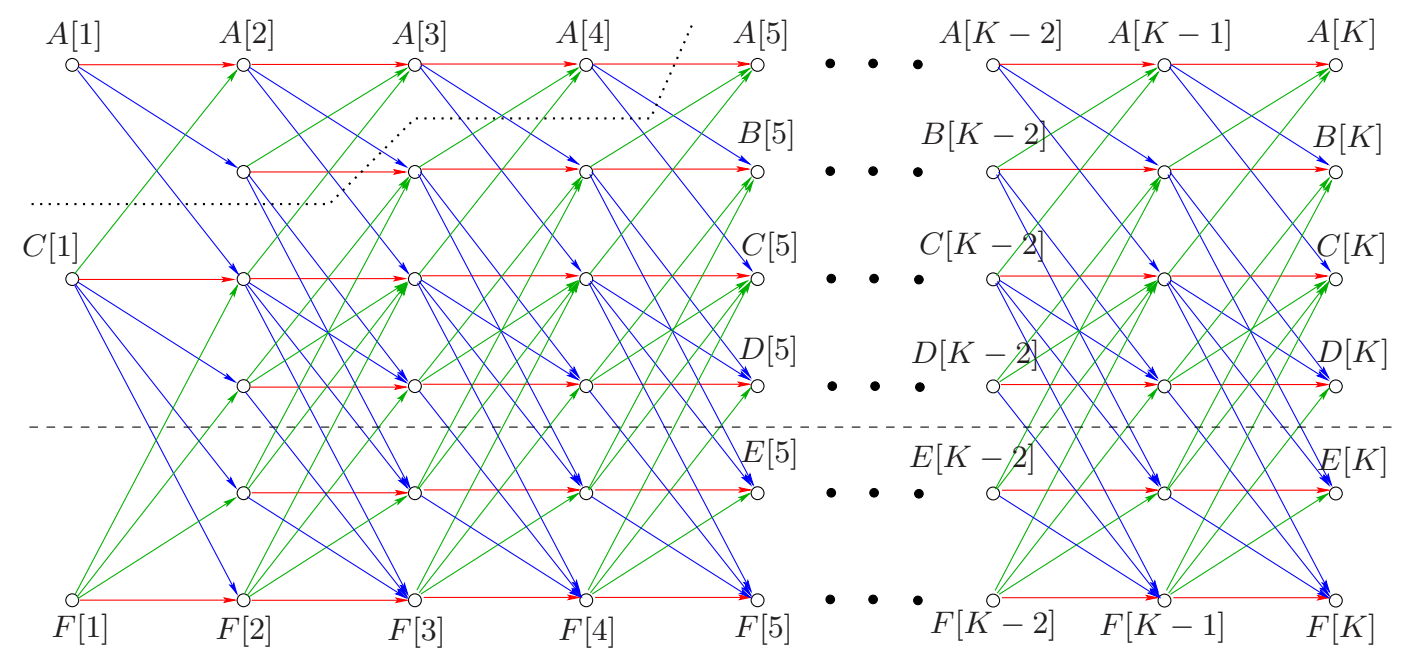

Fig. 2: Time expansion of the network in Figure 1, with an infinite value cut in dotted line, and a finite value cut in dash line.

However, its extension to arbitrary network is straight-forward. Note that the mapping functions described above, act exactly like normal mappings on the nodes of the time expansion network shown in Figure 2, where the horizontal links between different instances of the same node have infinite capacity, which capture the fact that nodes have infinite memory and are able to use all they have received in the previous blocks and map them to the next transmit sequences. At the end block $K$, each node $v \in \mathcal{S}$ has received $\mathbf{Y}_{v}=\left(\mathbf{y}_{v}^{(k)} ; k=1,2, \ldots, K\right)$. Let the source codewords $s_{A}^{M}(i), s_{C}^{M}(j)$, and $s_{F}^{M}(\ell)$ be sent. An errors at this node occurs if it cannot distinguish between $\left(s_{C}^{M}(j), s_{F}^{M}(\ell)\right)$ and $\left(s_{C}^{M}\left(j^{\prime}\right), s_{F}^{M}\left(\ell^{\prime}\right)\right)$ for some $\left(j^{\prime}, \ell^{\prime}\right) \neq$ $(j, \ell)$, since it already knows its own source sequence through the infinite capacity links. The error analysis for the time expansion network is exactly the same as that of the network in Section IV. Therefore, by considering the cuts and repeating a similar argument, one can show that the probability of error vanishes if

$$
H\left(\mathcal{S}_{\mathcal{J}} \mid \mathcal{S}_{\mathcal{J}^{c}}\right)<\mathcal{C}_{\text {time-exp }}(\mathcal{J}, v)
$$

for all $\mathcal{J} \subseteq \mathcal{S}$ and all $v \in \mathcal{J}^{c}$. It remains to show that the RHS of (26) is the same as the RHS of the condition in the theorem statement. Note that if a cut $\Omega$ satisfies $v[k] \in \Omega$ and $v\left[k^{\prime}\right] \in \Omega^{c}$, then the cut-value would be infinity, since it has to cut at least one horizontal link. Hence, the cuts with finite value, have to separate the nodes at different stages identically, and therefore, have an one-to-one correspondence with the cuts of the original network.

\section{REFERENCES}

[1] E. Perron, "Information-theoretic secrecy for wireless networks", Ph.D. dissertation, EPFL, Lausanne, 2009.

[2] T. M. Cover and J. A. Thomas, Elements of information theory, New York: Wiley, 1991.

[3] S. Avestimehr, S. N. Diggavi, and D. N. C. Tse, "Wireless network information flow," Proceedings of Allerton Conference on Communication, Control, and Computing, Illinois, September 2007.

[4] S. Avestimehr, S. N. Diggavi, and D. N. C. Tse, "Wireless network information flow: A aeterministic approach," IEEE Trans. Inform. Theory, 2009, submitted, http://arxiv.org/abs/0906.5394.

[5] T. Cover, A. El-Gamal and M. Salehi, "Multiple access channels with arbitrarily correlated sources," IEEE Trans. Inform. Theory, vol. 26, no. 2, pp. 648-657, November 1980.

[6] I. Csiszar and J. Körner, "Towards a general theory of source networks," IEEE Trans. Inform. Theory, vol. 26, no. 2, pp. 155-165, March 1980.

[7] P. Gàcs and J. Körner, "Common information is far less than mutual information," Problems of Control and Information Theory, vol. 2, no. 2, pp. 149-162, 1973.

[8] D. Gündüz, E. Erkip, A. Goldsmith, H. V. Poor, "Source and Channel Coding for Correlated Sources Over Multiuser Channels," IEEE Trans. Inform. Theory, vol. 55, no. 9, pp. 3927-3944, Sep. 2009.

[9] D. Slepian and J K. Wolf, "Noiseless coding of correlated information sources," IEEE Trans. Inform. Theory, vol. 19, no. 4, pp. 471-480, July 1973.

[10] S. B. Korada and D. Vasudevan, "Broadcast and Slepian-Wolf Multicast over Aref Networks," IEEE Int. Symp. on Information Theory (ISIT), Toronto, Canada, July, 2008.

[11] S. Mohajer, C. Tian, and S. N. Diggavi, "On source transmission over deterministic relay networks," EPFL Technical Report, Available at http://infoscience.epfl.ch 